\title{
The elusive excited states of bithiophene: a CASPT2 detective story
}

\author{
Marcin Andrzejak · Henryk A. Witek
}

Received: 1 March 2011 / Accepted: 3 March 2011 / Published online: 20 March 2011

(C) The Author(s) 2011. This article is published with open access at Springerlink.com

\begin{abstract}
A systematic multi-reference perturbation theory investigation of the excitation energies and oscillator strengths for the lowest excited states of 2,2'-bithiophene unequivocally shows that its optical spectrum is produced by two ${ }^{1} B_{u}$ states separated from each other by approximately $1 \mathrm{eV}$. This picture is confirmed by additional calculations with alternative quantum chemical methods. Our findings are in strong contrast with the previous CASPT2 results of Rubio et al. [J Chem Phys 102:3580 (1995) and Chem Phys Chem 4:1308 (2003)], who predicted that the two lowest ${ }^{1} B_{u}$ states are quasi-degenerate. The methodological reasons responsible for the previous seemingly erroneous assignment of the optical spectrum of bithiophene are identified and explained in terms of unusually large coupling between the ${ }^{1} B_{u}$ states introduced by dynamical correlation effects. A general discussion of applicable computational techniques is offered aiming at avoiding similar problems for other molecular systems.
\end{abstract}

Keywords Excited states - Bithiophene - CASSCF . CASPT2 - Vertical excitation energies · Absorption spectrum

Electronic supplementary material The online version of this article (doi:10.1007/s00214-011-0916-x) contains supplementary material, which is available to authorized users.

M. Andrzejak ( $\square)$

Department of Theoretical Chemistry, Faculty of Chemistry, Jagiellonian University, Ingardena 3, 30-060 Kraków, Poland e-mail: andrzeja@chemia.uj.edu.pl

H. A. Witek

Department of Applied Chemistry and Institute of Molecular Science, National Chiao Tung University, Hsinchu, Taiwan e-mail: hwitek@mail.nctu.edu.tw

\section{Introduction}

Spectroscopy of 5-membered heterocyclic rings has been extensively studied for several decades [1-15]. Excited states of thiophene attracted particular attention as this molecule constitutes the smallest building block of oligo and polythiophenes-materials extensively used in organic electronics [16-20]. In consequence, the energetics and electronic structure of thiophene are quite firmly established. The situation is different for 2,2'-bithiophene (hereafter referred to as bithiophene or $2 \mathrm{~T}$ ), mostly due to the relatively large size of the molecule. It is, however, well documented [21-23] that the adiabatic energy of the lowest, dipole-allowed excitation is located at 3.83-3.88 $\mathrm{eV}$. Room temperature gas-phase [23] and solution spectra [24] revealed an intensive band with oscillator strength $f=0.29$ located just above $4 \mathrm{eV}$ and a weaker one with $f=0.13$, at about $5 \mathrm{eV}$. These transitions were tentatively classified as $\mathrm{X}^{1} A_{g} \rightarrow 1{ }^{1} B_{u}$ and $\mathrm{X}^{1} A_{g} \rightarrow 2{ }^{1} B_{u}$, respectively, assuming planar, $C_{2 h}$ conformation of trans-bithiophene (Fig. 1). Another low-intensity band of $2 \mathrm{~T}$ was tentatively located above $6 \mathrm{eV}$.

Bithiophene can be regarded as a challenging system also for quantum chemistry because of its non-planarity and very flat torsional potential in its ground state. It is perhaps the reason that there have been only few theoretical studies dealing with the excited states of bithiophene. Rubio et al. [25, 26] applied the CASSCF/CASPT2 methodology to study the lowest $\pi-\pi^{*}$ excitations of $2 \mathrm{~T}$. They reported a pair of $B_{u}$ states of similar energies (3.88 and $4.15 \mathrm{eV}$ ) and a third state of considerably higher energy $(5.53 \mathrm{eV})$. The same excitations computed for trans-gauche conformation of bithiophene (optimized for the ground state at the MP2 level of theory) yielded slightly higher energies of 4.22, 4.36, and $5.79 \mathrm{eV}$. The agreement 


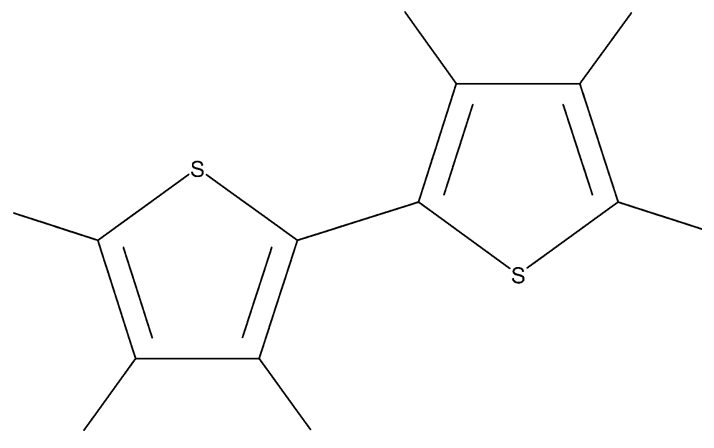

Fig. 1 Molecule of 2,2'-bithiophene

with experimental findings was considered quite satisfactory, which might indicate that our understanding of the lowest excited states of bithiophene is thorough.

The theoretical prediction of a pair of quasi-degenerate states is neither confirmed nor ruled-out by the existing experimental data. However, the fact that the two lowest, optically allowed excited singlet states of $2 \mathrm{~T}$ belong to the same irreducible representation and simultaneously have nearly degenerate energies of about $4 \mathrm{eV}$ is somewhat disquieting. Both of these states originate from the $\pi-\pi^{*}$ excitations. One would intuitively expect a strong coupling between the corresponding wave functions, leading to their considerable mixing, and consequently, to a substantial energy separation between the resulting states. Such an effect could be missing if the states originate from different types of excitations (e.g., $\pi-\pi^{*}$ and $n-\pi^{*}$ ), but this does not seem to be the case for 2T. An analysis of the $1{ }^{1} B_{u}$ and $2{ }^{1} B_{u}$ CASSCF wave functions suggests close similarity between both states. The major configuration $(43 \%)$ in the $1^{1} B_{u}$ wave function originates from the HOMO $\rightarrow$ LUMO excitation; there are also sizable additions of the HOMO $2 \rightarrow$ LUMO $(20 \%)$ and HOMO $\rightarrow$ LUMO +2 (7\%) excitations. The $2^{1} B_{u}$ wave function structure is strikingly similar, with the contributions of the respective configurations equal to 36,19 , and $9 \%$.

Is it possible that the quasi-degeneracy of the two lowest excited states of $2 \mathrm{~T}$ is merely an artifact of the applied computational methodology? The quoted results of Rubio et al. were computed using single-state CASPT2 formalism in conjunction with state-averaged CASSCF orbitals, the $\pi$-only valence active space, and relatively small atomic basis sets of double- $\zeta$ quality. This means that there is plenty of room for possible improvement of the theoretical treatment with respect to the methodological parameters: choice of the one-electron basis, definition of the manyelectron basis, estimating the effect of state-averaging, accounting for the dynamical correlation mixing between both wave functions, etc. Another aspect that is worth a careful inspection concerns the presence of intruder states in the CASPT2 treatment. Our recent work [27, 28] shows that spectroscopic and molecular parameters computed with multi-reference perturbation theory using shift technique may heavily depend on the magnitude of the shift parameter in situations when the calculations are plagued by severe intruder states [29]. Taking into account all the issues raised here, we feel that there exists a need for careful inspection of the previous theoretical results and testing various assumptions made in the previous work in order to give a final interpretation of the experimentally observed optical spectrum of $2 \mathrm{~T}$.

The natural way to verify the findings of Rubio et al. would be to apply the multi-state version of the CASPT2 method, which allows for coupling of the correlated states via the offdiagonal elements of the effective CASPT2 Hamiltonian. Usually, the off-diagonal dynamical correlation is small and does not introduce large changes to the energy spectrum. Such a physical picture is expected since most of the interactions between the states is supposed to be accounted for by the static correlation at the CASSCF level. The situation, however, may be different in cases of nearly degenerate excited states, which can be strongly mixed even by small off-diagonal coupling. The off-diagonal coupling may introduce considerable changes also in situations when the CASSCF description of the excited states is inadequate, which is signalized by unusually large offdiagonal elements of the effective Hamiltonian. Clearly, nonvanishing off-diagonal elements of the CASPT2 Hamiltonian in Rubio's calculations could cause additional splittings and lead to a completely different description of the low-lying electronic states of bithiophene.

The primary goal of this work is to investigate the excitation energies and oscillator strengths of the lowest singlet states of bithiophene using a series of computational approaches in order to obtain unequivocal assignment of the observed experimental optical spectra. To this end, we extend the previously applied single-state CASSCF/CASPT2 treatment of Rubio et al. by using a wider selection of basis sets and a variety of active spaces. Additionally, we perform the multi-state CASPT2 calculations to verify the interpretational problems found in Rubio's results. Finally, we compare the results of our extended CASPT2 calculations with analogous results obtained by other quantum chemical methods. The structure of this paper is as follows. Section 2 gives detailed information about available experimental results on the low-lying singlet states of $2 \mathrm{~T}$. Section 3 summarizes the details of computational techniques employed. Section 4 constitutes the main body of this paper, giving the analysis and discussion of the obtained results. Finally, Sect. 5 contains the conclusions and lists the most important findings of our work.

\section{Experimental evidence}

Owing to the size of the molecule, conclusive experimental data concerning excited states of bithiophene are by far 
scarcer than for thiophene or other 5-membered heterocyclic rings. The supersonic-jet [21] and the photodetachment photoelectron spectra [22] show that the gas-phase adiabatic excitation energy for the lowest dipole-allowed state $\left(\mathrm{X}{ }^{1} A_{g} \rightarrow 1{ }^{1} B_{u}\right.$ ) can be quite firmly set at $3.86-3.88 \mathrm{eV}$. A slightly lower value of $3.83 \mathrm{eV}$, based on the room temperature absorption spectrum of bithiophene vapors, was reported by Bellêtete et al. [23]. This spectrum shows an intense, broad band centered at $4.31 \mathrm{eV}$ and a weaker band with maximum at $5.08 \mathrm{eV}$. These energies can be tentatively adopted for vertical energies of the lowest dipole-allowed transitions of $2 \mathrm{~T}$.

A delicate point concerning the comparison of the experimental energies with the calculated ones is associated with the torsional potential for the ground and the lowest excited states of $2 \mathrm{~T}$. The potential for the ground state is known to possess a double minimum around the trans-planar conformation, with the equilibrium structure corresponding to the dihedral angle of about $150^{\circ}$. The corresponding energy barrier is only $0.01-0.04 \mathrm{eV}$. [23, 24, 30-33] By contrast, in the lowest excited state, $2 \mathrm{~T}$ has been found to be trans-planar with a deep, steep single minimum on the potential energy surface (PES) [23, 24]. One should thus expect that if the $2 \mathrm{~T}$ molecule assumed the planar conformation, the lowest vertical absorption energy would be considerably lower than the gas-phase value, whereas the adiabatic energy would be reduced only marginally. Such a behavior was indeed observed by Becker et al. [24], who measured absorption spectra of $2 \mathrm{~T}$ in dioxane in $298 \mathrm{~K}$ and in $77 \mathrm{~K}$. The band center underwent a red shift of $0.12 \mathrm{eV}$ when the temperature was lowered, and at the same time, the energy of the $0-0$ line was decreased only by $0.025 \mathrm{eV}$. Becker et al. attributed these changes to the planarization of $2 \mathrm{~T}$ enforced by freezing of the solvent. Accordingly, the vertical energy of the $\mathrm{X}^{1} A_{g} \rightarrow{ }^{1} B_{u}$ excitation, calculated for the planar trans conformation of $2 \mathrm{~T}$, ought to be compared to $4.19 \mathrm{eV}$, which is the experimental gasphase value corrected by the shift inferred from the $2 \mathrm{~T}$ dioxane spectra. Note, however, that according to Rubio et al. the lowenergy absorption band is formed by two unresolved excitations to closely spaced ${ }^{1} B_{u}$ states. If it is the case, the experimental value of $4.31 \mathrm{eV}$ has to be regarded only as an effective value for two overlapping bands, and the temperature dependence of the absorption spectra described above may have an alternative explanation.

Following the discussion in the last two Sections, we assume for the rest of this paper that the experimental vertical excitation energies for planar $2 \mathrm{~T}$ are $4.19 \mathrm{eV}$ for $\mathrm{X}^{1} A_{g} \rightarrow 1{ }^{1} B_{u}$ and $5.08 \mathrm{eV}$ for $\mathrm{X}^{1} A_{g} \rightarrow 2{ }^{1} B_{u}$. Consequently, the energy gap between both excited states is $0.89 \mathrm{eV}$. The corresponding oscillator strengths of both transitions are 0.29 and 0.13 .

\section{Computational details}

The vertical excitation energies for the two lowest ${ }^{1} B_{u}$ states of $2 \mathrm{~T}$ were computed using the second-order singleand multi-state complete active space perturbation theory (CASPT2) with the IPEA shift of $0.25 \mathrm{eV}$ and the imaginary shift of 0.1 hartree as implemented in the MOLCAS 7.4 package of programs [34]. The calculations were performed using a set of correlation-consistent basis sets of Dunning: cc-pVDZ, aug-cc-pVDZ, cc-pVTZ, and aug-ccpVTZ [35-37]. The zeroth-order wave functions were optimized at the complete active space self-consistent field (CASSCF) level. The CASSCF calculations for the ground state $\left(\mathrm{X}^{1} A_{g}\right)$ were performed state-specifically, while for the excited states, state averaging with the ratio 1:1 was performed for $1{ }^{1} B_{u}$ and $2{ }^{1} B_{u}$. The effect of state-averaging was assessed by performing additional, state-specific CASSCF calculation for the $1{ }^{1} B_{u}$ state alone and projecting the resultant wave function onto its state-averaged analog. The vertical excitation energies and the corresponding oscillator strengths (determined with the CASSCF wave functions) were computed using the equilibrium, trans-planar $\left(C_{2 h}\right)$ geometrical structures of $2 \mathrm{~T}$ in its ground state optimized at the CASPT2 level using the valence (0550) active space. The resulting CASPT2/0550 equilibrium geometries for each of the employed here basis sets are given in auxiliary materials.

The subsequent CASPT2 calculations of the vertical excitation energies were performed using a variety of active spaces. Their choice was dictated by the following facts. Simple orbital analysis of bithiophene in its transplanar conformation leads to a general conclusion that the lowest valence electronic states belong either to $A_{g}$ or $B_{u}$ irreducible representations. The lowest excited states originate from the $\pi-\pi^{*}$ excitations; the $n-\pi^{*}$ and $\sigma-\pi^{*}$ transitions are located much higher in energy. Therefore, the natural choice of the active space for $2 \mathrm{~T}$ comprises of ten valence $\pi$ orbitals (five $b_{g}$ and five $a_{u}$ ) and 12 active electrons. We denote this active space briefly as 0550 , where the consecutive numbers refer to the number of active orbitals in the following irreducible representations: $a_{g}, b_{g}, a_{u}$, and $b_{u}$, respectively. Note that this active space is identical to that used previously by Rubio et al. It was pointed out by Pastore et al. that some of the lowest $\pi-\pi^{*}$ excitation energies calculated for 5-membered heterocyclic rings (pyrrole, furan, and thiophene), [6, 11, 15] are strongly sensitive to the presence of additional, virtual $\pi$ orbitals in the active space. Therefore, we extended the natural valence active space by adding to it additional virtual $\pi$ orbitals. The resulting extended complete active spaces can be briefly denoted as 0570,0660 , and 0770 . The results obtained with the augmented basis sets showed considerably higher degree of sensitivity to the choice of the active space. To understand this dependence better, we have additionally 
investigated a number of auxiliary active spaces: 0560, 0580, 0650, 0670, 0680, 0750, 0760, and 0780.

\section{Results}

\subsection{Single-state CASPT2 results}

The single-state CASPT2 excitation energies and the corresponding CASSCF oscillator strengths computed using the cc-pVDZ and cc-pVTZ basis sets are presented in Table 1. The CASSCF energies of both transitions appear to be only weakly dependent on the choice of the active space or the basis set. The CASSCF energy separation between the two excited states ranges from 0.27 to $0.44 \mathrm{eV}$. Different performance of the two basis sets becomes evident when the dynamic correlation effects are included. In the cc-pVDZ basis sets, the two ${ }^{1} B_{u}$ states are almost quasi-degenerate, while in the cc-pVTZ basis set, the CASPT2 energy separations are similar to those computed with CASSCF. Moreover, the CASPT2 corrections calculated with the cc-pVDZ basis set are considerably larger for the $2{ }^{1} B_{u}$ state leading to a reversed energy ordering of the two ${ }^{1} B_{u}$ states at the CASPT2 level. It is evident from this discussion that the basis set effect is large, and the quasi-degeneracy of two low-lying ${ }^{1} B_{u}$ states obtained earlier by Rubio et al. in a basis set of double- $\zeta$ quality seems to be at least disputable. As the reader will be able to see from the following paragraphs, this controversy is further supported by other results.

The single-state CASPT2 excitation energies and the corresponding CASSCF oscillator strengths computed using the aug-cc-pVDZ and aug-cc-pVTZ basis sets are presented in Tables 2 and 3, respectively. These results in general tend to confirm the picture obtained with the cc-pVTZ basis set and are in contrast to the data obtained

Table 1 State-average CASSCF/single-state CASPT2 excitation energies (in eV) and oscillator strengths for transitions to two lowest $\mathrm{B}_{\mathrm{u}}$ singlet states of $2 \mathrm{~T}$, obtained with the non-augmented basis sets, and selected active spaces

\begin{tabular}{|c|c|c|c|c|c|c|c|c|c|}
\hline \multirow{2}{*}{$\begin{array}{l}\text { Basis } \\
\text { set }\end{array}$} & \multirow{2}{*}{$\begin{array}{l}\text { Active } \\
\text { space }\end{array}$} & \multicolumn{3}{|l|}{$1^{1} \mathrm{~B}_{\mathrm{u}}$} & \multicolumn{3}{|l|}{$2^{1} B_{u}$} & \multirow{2}{*}{$\begin{array}{l}\Delta \mathrm{E} \\
\mathrm{CASSCF}\end{array}$} & \multirow{2}{*}{$\begin{array}{l}\Delta \mathrm{E} \\
\mathrm{CASPT} 2\end{array}$} \\
\hline & & $\mathrm{E}_{\mathrm{CASSCF}}$ & $\begin{array}{l}\mathrm{E}_{\mathrm{CASPT} 2} / \\
\text { (osc. strength) }\end{array}$ & $\begin{array}{l}\text { CASPT2 } \\
\text { correction }\end{array}$ & $\mathrm{E}_{\mathrm{CAS}}$ & $\begin{array}{l}\mathrm{E}_{\mathrm{CASPT} 2} / \\
\text { (osc. strength) }\end{array}$ & $\begin{array}{l}\text { CASPT2 } \\
\text { correction }\end{array}$ & & \\
\hline \multirow[t]{4}{*}{ cc-pVDZ } & 0550 & 5.69 & $4.76(0.23)$ & 0.93 & 6.13 & $4.72(0.39)$ & 1.41 & 0.44 & -0.04 \\
\hline & 0570 & 5.63 & $4.77(0.23)$ & 0.86 & 6.01 & $4.76(0.39)$ & 1.25 & 0.38 & -0.01 \\
\hline & 0660 & 5.71 & $4.89(0.16)$ & 0.83 & 6.07 & $4.56(0.41)$ & 1.51 & 0.36 & -0.33 \\
\hline & 0770 & 5.64 & $4.78(0.20)$ & 0.86 & 6.01 & $4.72(0.38)$ & 1.29 & 0.37 & -0.06 \\
\hline \multirow[t]{4}{*}{ cc-pVTZ } & 0550 & 5.73 & $4.40(0.30)$ & 1.33 & 6.15 & $4.83(0.31)$ & 1.32 & 0.42 & 0.43 \\
\hline & 0570 & 5.63 & $4.38(0.32)$ & 1.25 & 6.00 & $4.94(0.27)$ & 1.06 & 0.37 & 0.56 \\
\hline & 0660 & 5.76 & $4.48(0.25)$ & 1.29 & 6.03 & $4.73(0.30)$ & 1.30 & 0.27 & 0.25 \\
\hline & 0770 & 5.64 & $4.39(0.28)$ & 1.25 & 5.98 & $4.91(0.25)$ & 1.07 & 0.34 & 0.52 \\
\hline
\end{tabular}

Table 2 State-average CASSCF/single-state CASPT2 excitation energies (in eV) and oscillator strengths for transitions to two lowest $\mathrm{B}_{\mathrm{u}}$ singlet states of 2T, obtained with the aug-cc-pVDZ basis set, and a series of active spaces

\begin{tabular}{|c|c|c|c|c|c|c|c|c|}
\hline \multirow{2}{*}{$\begin{array}{l}\text { Active } \\
\text { space }\end{array}$} & \multicolumn{3}{|l|}{$1^{1} B_{u}$} & \multicolumn{3}{|l|}{$2^{1} \mathrm{~B}_{\mathrm{u}}$} & \multirow{2}{*}{$\begin{array}{l}\Delta \mathrm{E} \\
\mathrm{CASSCF}\end{array}$} & \multirow{2}{*}{$\begin{array}{l}\Delta \mathrm{E} \\
\mathrm{CASPT} 2\end{array}$} \\
\hline & $\mathrm{E}_{\mathrm{CASSCF}}$ & $\begin{array}{l}\mathrm{E}_{\text {CASPT2 }} / \\
\text { (osc. strength) }\end{array}$ & $\begin{array}{l}\text { CASPT2 } \\
\text { correction }\end{array}$ & $\mathrm{E}_{\mathrm{CAS}}$ & $\begin{array}{l}\mathrm{E}_{\text {CASPT2 }} / \\
\text { (osc. strength) }\end{array}$ & $\begin{array}{l}\text { CASPT2 } \\
\text { correction }\end{array}$ & & \\
\hline 0550 & 5.63 & $4.36(0.27)$ & 1.27 & 6.02 & $4.64(0.32)$ & 1.38 & 0.39 & 0.28 \\
\hline 0560 & 5.54 & $4.10(0.31)$ & 1.44 & 5.84 & $5.00(0.19)$ & 0.83 & 0.30 & 0.90 \\
\hline 0570 & 5.45 & $4.15(0.32)$ & 1.30 & 5.79 & $4.98(0.20)$ & 0.81 & 0.34 & 0.83 \\
\hline 0580 & 5.40 & $4.13(0.33)$ & 1.26 & 5.73 & $4.98(0.19)$ & 0.76 & 0.34 & 0.85 \\
\hline 0650 & 5.60 & $4.35(0.25)$ & 1.25 & 6.02 & $4.63(0.31)$ & 1.38 & 0.42 & 0.28 \\
\hline 0660 & 5.62 & $4.05(0.29)$ & 1.58 & 5.86 & $4.94(0.18)$ & 0.92 & 0.23 & 0.89 \\
\hline 0670 & 5.46 & $4.22(0.27)$ & 1.24 & 5.80 & $4.87(0.22)$ & 0.92 & 0.34 & 0.65 \\
\hline 0680 & 5.38 & $4.17(0.30)$ & 1.21 & 5.78 & $4.92(0.21)$ & 0.86 & 0.37 & 0.75 \\
\hline 0750 & 5.55 & $4.28(0.27)$ & 1.28 & 5.98 & $4.77(0.26)$ & 1.21 & 0.43 & 0.49 \\
\hline 0760 & 5.54 & $4.06(0.31)$ & 1.49 & 5.86 & $5.00(0.16)$ & 0.86 & 0.32 & 0.94 \\
\hline 0770 & 5.47 & $4.11(0.29)$ & 1.36 & 5.83 & $4.97(0.17)$ & 0.86 & 0.36 & 0.86 \\
\hline 0780 & 5.39 & $4.12(0.31)$ & 1.27 & 5.71 & $5.01(0.17)$ & 0.69 & 0.32 & 0.89 \\
\hline
\end{tabular}


Table 3 State-average CASSCF/single-state CASPT2 excitation energies (in eV) and oscillator strengths for transitions to two lowest $\mathrm{B}_{\mathrm{u}}$ singlet states of $2 \mathrm{~T}$, obtained with the aug-cc-pVTZ basis set, and a series of active spaces

\begin{tabular}{|c|c|c|c|c|c|c|c|c|}
\hline \multirow{2}{*}{$\begin{array}{l}\text { Active } \\
\text { space }\end{array}$} & \multicolumn{3}{|l|}{$1^{1} B_{u}$} & \multicolumn{3}{|l|}{$2{ }^{1} \mathrm{~B}_{\mathrm{u}}$} & \multirow{2}{*}{$\begin{array}{l}\Delta \mathrm{E} \\
\mathrm{CASSCF}\end{array}$} & \multirow{2}{*}{$\begin{array}{l}\Delta \mathrm{E} \\
\mathrm{CASPT} 2\end{array}$} \\
\hline & $\mathrm{E}_{\mathrm{CASSCF}}$ & $\begin{array}{l}\mathrm{E}_{\mathrm{CASPT} 2} / \\
\text { (osc. strength) }\end{array}$ & $\begin{array}{l}\text { CASPT2 } \\
\text { correction }\end{array}$ & $\mathrm{E}_{\mathrm{CAS}}$ & $\begin{array}{l}\mathrm{E}_{\mathrm{CASPT} 2} / \\
\text { (osc. strength) }\end{array}$ & $\begin{array}{l}\text { CASPT2 } \\
\text { correction }\end{array}$ & & \\
\hline 0550 & 5.69 & $4.19(0.32)$ & 1.50 & 6.11 & $4.85(0.27)$ & 1.26 & 0.42 & 0.66 \\
\hline 0560 & 5.55 & $4.08(0.34)$ & 1.47 & 5.95 & $5.17(0.15)$ & 0.78 & 0.40 & 1.09 \\
\hline 0570 & 5.48 & $4.14(0.33)$ & 1.34 & 5.90 & $5.15(0.17)$ & 0.75 & 0.42 & 1.01 \\
\hline 0580 & 5.41 & $4.12(0.34)$ & 1.29 & 5.84 & $5.12(0.16)$ & 0.71 & 0.43 & 1.00 \\
\hline 0650 & 5.66 & $4.20(0.29)$ & 1.46 & 6.10 & $4.81(0.26)$ & 1.29 & 0.44 & 0.61 \\
\hline 0660 & 5.66 & $3.99(0.32)$ & 1.67 & 5.98 & $5.10(0.14)$ & 0.87 & 0.31 & 1.11 \\
\hline 0670 & 5.50 & $4.17(0.29)$ & 1.32 & 5.89 & $5.08(0.18)$ & 0.81 & 0.39 & 0.91 \\
\hline 0680 & 5.41 & $4.17(0.31)$ & 1.24 & 5.87 & $5.08(0.17)$ & 0.80 & 0.47 & 0.91 \\
\hline 0750 & 5.62 & $4.15(0.32)$ & 1.47 & 6.07 & $4.90(0.22)$ & 1.17 & 0.45 & 0.75 \\
\hline 0760 & 5.60 & $4.01(0.33)$ & 1.59 & 5.96 & $5.13(0.13)$ & 0.84 & 0.37 & 1.12 \\
\hline 0770 & 5.51 & $4.10(0.31)$ & 1.42 & 5.94 & $5.10(0.15)$ & 0.83 & 0.42 & 1.00 \\
\hline 0780 & 5.41 & $4.11(0.32)$ & 1.29 & 5.81 & $5.14(0.13)$ & 0.67 & 0.41 & 1.03 \\
\hline
\end{tabular}

with the cc-pVDZ basis set. The results obtained with the cc-pVTZ basis set show mediocre agreement with experiment. In general, the energy separation between the $1{ }^{1} B_{u}$ and $2{ }^{1} B_{u}$ states is too small, and the intensity pattern of both transitions does not match the experimental picture. Including the diffuse functions in the basis set allows for substantial improvement of the calculated results in comparison with experiment. The CASPT2 excitation energies obtained with the augmented basis sets are substantially lowered for the $1{ }^{1} B_{u}$ state and slightly increased for the $2{ }^{1} B_{u}$ state. Consequently, the energy splitting between the two excited states becomes much larger. Also, the calculated oscillator strengths reveal considerable intensity shift in favor of the lower transition. The $\mathrm{X}^{1} A_{g} \rightarrow 1{ }^{1} B_{u}$ excitation energies are confined to a narrow range of $3.99-4.22 \mathrm{eV}$ (experimentally $\approx 4.19 \mathrm{eV}$ ) and the $\mathrm{X}^{1} A_{g} \rightarrow 2{ }^{1} B_{u}$ excitation energies, to $4.87-5.17 \mathrm{eV}$ (experimentally $\approx 5.08 \mathrm{eV}$ ). The calculated oscillator strength for the lower transition, equal to $0.27-0.34$, is in excellent agreement with the experimental value of 0.29 . The intensity of the higher excitation is somewhat overestimated $(0.13-0.22)$, as compared to the experimental value of 0.13. [24] Note that the results obtained with the largest studied here basis set, aug-cc-pVTZ, allow for the best reproduction of the experimental findings.

The results obtained with augmented basis sets show more pronounced dependence on the composition of the active space. To exploit the character of this dependence in detail, we carried out a more detailed study involving 12 different variants of the active space (see Tables 2 and 3, and Fig. 2 for details). The CASSCF excitation energies are reduced upon addition of extra $a_{u}$ orbitals to the active space, while they are relatively insensitive when extra $b_{g}$
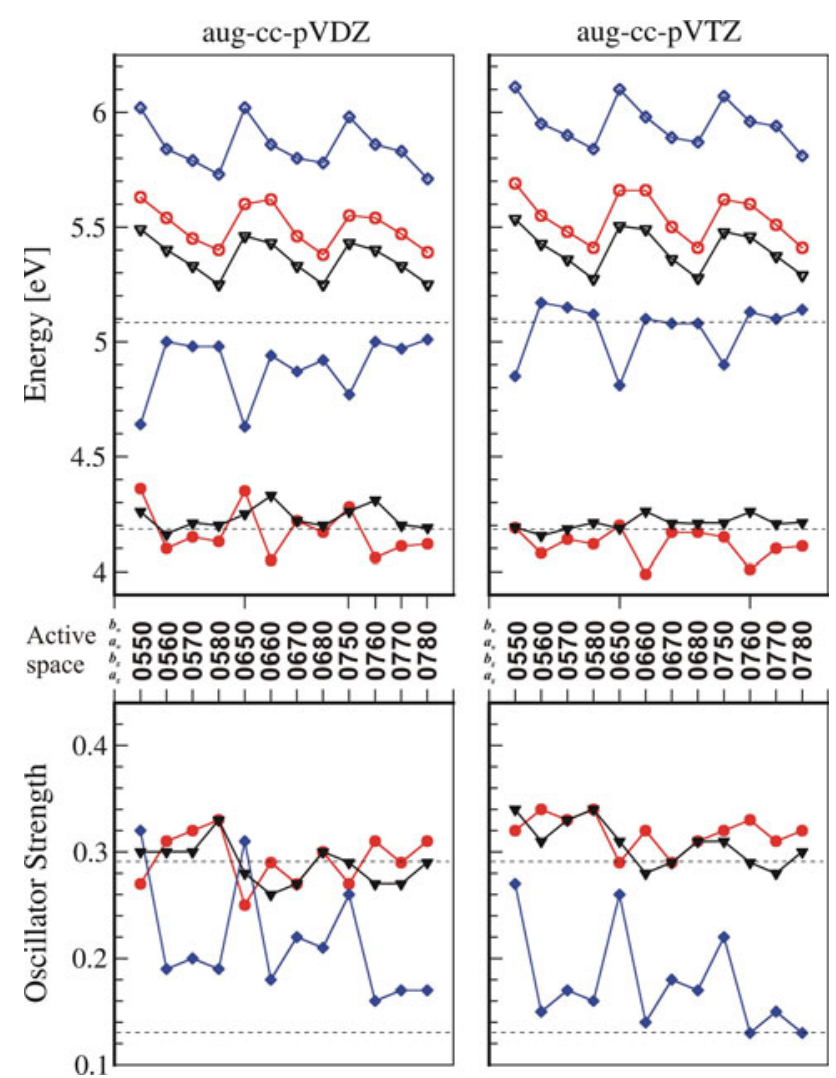

Fig. 2 The $\mathrm{X}{ }^{1} A_{g} \rightarrow 1{ }^{1} B_{u}$ and $\mathrm{X}^{1} A_{g} \rightarrow 2{ }^{1} B_{u}$ excitation energies (upper panels) and oscillator strengths (lower panels) of $2 \mathrm{~T}$ computed using the single-state CASPT2 (filled symbols) and CASSCF (open symbols) methods, and a variety of active spaces. The results for the $1^{1} B_{u}$ state are depicted using red circles (state-averaged calculations) and black triangles (state-specific calculations) and the results for the $2{ }^{1} B_{u}$ state, using blue diamonds (state-averaged calculations). For the definition of the active spaces (in abscissas), see text. Broken lines indicate the relevant experimental values 
Table 4 Single-state versus multi-state CASPT2 results (on top of the state-average CASSCF wave functions) obtained with the cc-pVDZ and cc-pVTZ basis sets and selected active spaces. All the energies and coupling elements are given in eV

\begin{tabular}{|c|c|c|c|c|c|c|c|c|}
\hline \multirow[t]{2}{*}{ Basis set } & \multirow[t]{2}{*}{ Active space } & \multicolumn{3}{|c|}{ Single-state CASPT2 } & \multicolumn{4}{|c|}{ Multi-state CASPT2 } \\
\hline & & $1^{1} \mathrm{~B}_{\mathrm{u}}$ & $2^{1} B_{u}$ & $\Delta \mathrm{E}$ & $1^{1} \mathrm{~B}_{\mathrm{u}}$ & $2^{1} B_{u}$ & $\left|\begin{array}{r}e f f \\
\hat{H}_{12}\end{array}\right|$ & $\Delta \mathrm{E}$ \\
\hline \multirow[t]{4}{*}{ cc-pVDZ } & 0550 & $4.76(0.23)$ & $4.72(0.39)$ & -0.04 & $3.84(0.44)$ & $5.65(0.09)$ & 0.91 & 1.81 \\
\hline & 0570 & $4.77(0.23)$ & $4.76(0.39)$ & -0.01 & $3.88(0.45)$ & $5.64(0.09)$ & 0.88 & 1.76 \\
\hline & 0660 & $4.89(0.16)$ & $4.56(0.41)$ & -0.33 & $3.88(0.42)$ & $5.56(0.09)$ & 0.82 & 1.68 \\
\hline & 0770 & $4.78(0.20)$ & $4.72(0.38)$ & -0.07 & $3.90(0.42)$ & $5.59(0.08)$ & 0.85 & 1.69 \\
\hline \multirow[t]{4}{*}{ cc-pVTZ } & 0550 & $4.40(0.30)$ & $4.83(0.31)$ & 0.43 & $3.64(0.42)$ & $5.59(0.09)$ & 0.95 & 1.95 \\
\hline & 0570 & $4.38(0.32)$ & $4.94(0.27)$ & 0.56 & $3.75(0.42)$ & $5.57(0.09)$ & 0.86 & 1.82 \\
\hline & 0660 & $4.48(0.25)$ & $4.73(0.30)$ & 0.25 & $3.66(0.38)$ & $5.55(0.08)$ & 0.94 & 1.89 \\
\hline & 0770 & $4.39(0.28)$ & $4.91(0.25)$ & 0.52 & $3.77(0.39)$ & $5.53(0.07)$ & 0.84 & 1.76 \\
\hline
\end{tabular}

orbitals are added. For the active spaces without additional $a_{u}$ orbitals, i.e., 0550, 0650, and 0750, the computed CASPT2 excitation energies and oscillator strengths are distinctly different than for the remaining active spaces. Therefore, we treat 0550,0650 , and 0750 as outliers and do not consider them in the analysis of the results. A detailed discussion concerning the role and characteristics of the extra $a_{u}$ orbitals in the active space will be given in Sect. 5 .

It should be noted in passing that the $\mathrm{X}^{1} A_{g} \rightarrow 1{ }^{1} B_{u}$ excitation energies obtained from the state-specific calculations for the $1{ }^{1} B_{u}$ state are relatively insensitive to the choice of the active space, which agrees with the observation that the active space substitution effect in the state-averaged calculations is visible mainly for the $2{ }^{1} B_{u}$ state. The state-specific $1^{1} B_{u}$ wave function projected onto its state-averaged analog gives overlap larger than $95 \%$ for the both augmented basis sets and all choices of active spaces but 0660 and 0760 . These results demonstrate that the description of the $1{ }^{1} B_{u}$ state of $2 \mathrm{~T}$ is not strongly affected by the averaging of the orbitals.

Summarizing the results obtained in this Section, we can state that accurate reproduction of experimental energy gap between the two ${ }^{1} B_{u}$ states and the correct intensity pattern requires two key factors: i) augmenting the natural active space (0550) with at least one additional orbital of symmetry $a_{u}$, and ii) using basis sets with auxiliary diffuse functions. It is also recommended to use a basis set of preferably triple- $\zeta$ quality, as the results obtained with the cc-pVDZ basis set raise severe interpretational controversies and can be most likely regarded as computational artifacts. Note that in light of these results, the previous findings of Rubio et al. concerning two almost quasidegenerate ${ }^{1} B_{u}$ states computed with a double- $\zeta$ quality basis set seem to be erroneous.

\subsection{Multi-state CASPT2 results}

The results discussed in Sect. 4.1 were obtained with single-state CASPT2 formalism. Accordingly, the coupling between the $1{ }^{1} B_{u}$ and $2{ }^{1} B_{u}$ states was accounted for only at the CASSCF level. In most situations this is sufficient; possible exceptions may concern systems with quasi-degenerated electronic level or extraordinary large CASPT2 couplings between the considered states. In this paragraph, we show that the quasi-degeneracy of the $1{ }^{1} B_{u}$ and $2{ }^{1} B_{u}$ states obtained with the single-state CASPT2 formalism and the ccpVDZ basis set is removed if one uses two-state CASPT2 formalism. Moreover, the two-state CASPT2 results obtained with all the considered here basis sets and active spaces display high level of consistency giving similar estimates of the $\mathrm{X}^{1} A_{g} \rightarrow 1{ }^{1} B_{u}$ and $\mathrm{X}^{1} A_{g} \rightarrow 2{ }^{1} B_{u}$ excitation energies and oscillator strengths.

The multi-state CASPT2 excitation energies, the corresponding CASSCF oscillator strengths, and the magnitude of the off-diagonal coupling elements computed using the cc-pVDZ and cc-pVTZ basis sets are presented in Table 4. Analogous results obtained with the aug-cc-pVDZ and augcc-pVTZ basis sets are presented in Tables 5 and 6 , respectively, and in Fig. 3. The first, most surprising observation concerning the presented data is the unusually large magnitude of the off-diagonal CASPT2 coupling elements, $\widetilde{H}_{1,2}^{e f f}$, between the $1{ }^{1} B_{u}$ and $2{ }^{1} B_{u}$ states. Such large values suggest that the active spaces employed here are not flexible enough to describe efficiently the static correlation in both studied wave functions. The coupling is the largest $(0.91-0.98 \mathrm{eV})$ for the smallest active space studied here: 0550. In general, the coupling is reduced if the size of the active space and/or basis set is enlarged. The smallest coupling $(0.40 \mathrm{eV})$ is observed for the largest active space and basis set considered in this study (0780/ aug-cc-pVTZ), which furnish most variational freedom to account properly for the multi-reference character of both wave functions.

For the cc-pVDZ basis set, the magnitude of the offdiagonal coupling element exceeds vastly single-state CASPT2 separation energy between the both ${ }^{1} B_{u}$ states and 

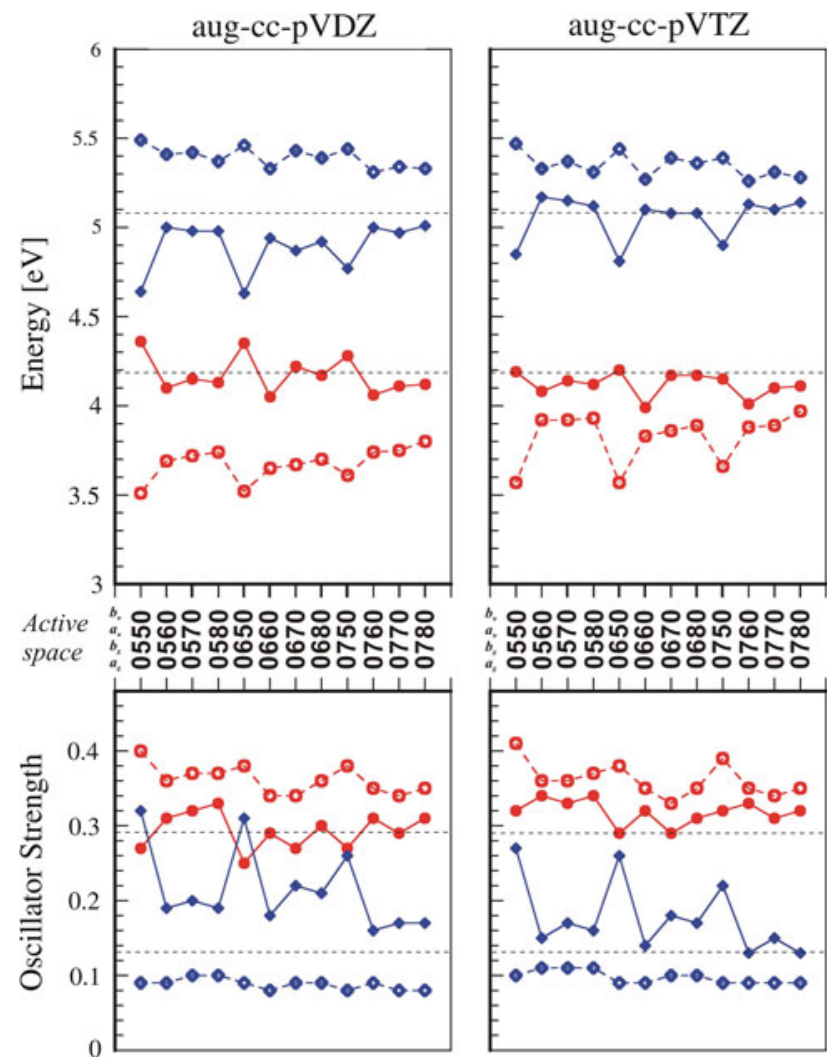

Fig. 3 The comparison between the single-state and multi-state CASPT2 excitation energies (upper panels) and oscillator strengths (lower panels) for the $1{ }^{1} B_{u}$ (red circles) and $2{ }^{1} B_{u}$ (blue diamonds) states of $2 \mathrm{~T}$ computed using various active spaces. The single-state results are depicted using filled symbols, and the multi-state results, using open symbols. For the definition of the active spaces (in abscissas), see text. Broken lines indicate the relevant experimental values brings about dramatic changes to the CASPT2 energies of the two states and to the character of their wave functions. The resulting splitting is now as large as $1.69-1.81 \mathrm{eV}$. A comparably vast energy gap between the $1{ }^{1} B_{u}$ and $2{ }^{1} B_{u}$ states is also found for other basis sets. The oscillator strengths show a similarly weak dependence on the choice of the basis set and active space, with lower transition being about 4-5 times more intense than the higher one for all the applied combinations of the methodological parameters. It is apparent that the differences of both energies and intensities obtained in the multi-state CASPT2 calculations substantially overestimate the experimental values. The best agreement with experiment is again obtained for the largest active space and basis set investigated here (0780/ aug-cc-pVTZ). The lower excited state, $1{ }^{1} B_{u}$, is located $3.97 \mathrm{eV}$ above the ground state (experimentally $\approx$ $4.19 \mathrm{eV})$ and the higher excited state, $2{ }^{1} B_{u}, 5.28 \mathrm{eV}$ higher than $\mathrm{X}^{1} A_{g}$ (experimentally $\left.\approx 5.08 \mathrm{eV}\right)$. The computed oscillator strengths of the corresponding transitions $(0.35$ and 0.09 , respectively) follow the experimental intensity pattern (0.29 and 0.13). Even if the positions of both excited states and the intensities of associated transitions are reproduced quite well, the resulting energy splitting $(1.31 \mathrm{eV})$ is still overestimated by almost $50 \%$ in comparison with the experimental value $(0.89 \mathrm{eV})$.

It is quite surprising that it is possible to obtain much better agreement with experiment using the single-state rather than the multi-state CASPT2 formalism. One of the possible explanations for this behavior can be overestimation of dynamical correlation by CASPT2. It is well known that the second-order perturbation theory (PT) usually overshoots the correlation energy and the third-

Table 5 Single-state versus multi-state CASPT2 results (on top of the state-average CASSCF wave functions) obtained with the aug-cc-pVDZ and a series of active spaces. All the energies and coupling elements are given in $\mathrm{eV}$

\begin{tabular}{|c|c|c|c|c|c|c|c|}
\hline \multirow{2}{*}{$\begin{array}{l}\text { Active } \\
\text { space }\end{array}$} & \multicolumn{3}{|c|}{ Single-state CASPT2 } & \multicolumn{4}{|c|}{ Multi-state CASPT2 } \\
\hline & $1^{1} \mathrm{~B}_{\mathrm{u}}$ & $2^{1} \mathrm{~B}_{\mathrm{u}}$ & $\Delta \mathrm{E}$ & $1^{1} \mathrm{~B}_{\mathrm{u}}$ & $2^{1} \mathrm{~B}_{\mathrm{u}}$ & $\left|\begin{array}{r}e f f \\
H_{12}\end{array}\right|$ & $\Delta \mathrm{E}$ \\
\hline 0550 & $4.36(0.27)$ & $4.64(0.32)$ & 0.28 & $3.51(0.40)$ & $5.49(0.09)$ & 0.98 & 1.98 \\
\hline 0560 & $4.10(0.31)$ & $5.00(0.19)$ & 0.90 & $3.69(0.36)$ & $5.41(0.09)$ & 0.73 & 1.72 \\
\hline 0570 & $4.15(0.32)$ & $4.98(0.20)$ & 0.83 & $3.72(0.37)$ & $5.42(0.10)$ & 0.74 & 1.70 \\
\hline 0580 & $4.13(0.33)$ & $4.98(0.19)$ & 0.85 & $3.74(0.37)$ & $5.37(0.10)$ & 0.70 & 1.63 \\
\hline 0650 & $4.35(0.25)$ & $4.63(0.31)$ & 0.28 & $3.52(0.38)$ & $5.46(0.09)$ & 0.96 & 1.94 \\
\hline 0660 & $4.05(0.29)$ & $4.94(0.18)$ & 0.89 & $3.65(0.34)$ & $5.33(0.08)$ & 0.72 & 1.68 \\
\hline 0670 & $4.22(0.27)$ & $4.87(0.22)$ & 0.65 & $3.67(0.34)$ & $5.43(0.09)$ & 0.82 & 1.76 \\
\hline 0680 & $4.17(0.30)$ & $4.92(0.21)$ & 0.75 & $3.70(0.36)$ & $5.39(0.09)$ & 0.75 & 1.69 \\
\hline 0750 & $4.28(0.27)$ & $4.77(0.26)$ & 0.49 & $3.61(0.38)$ & $5.44(0.08)$ & 0.88 & 1.84 \\
\hline 0760 & $4.06(0.31)$ & $5.00(0.16)$ & 0.94 & $3.74(0.35)$ & $5.31(0.09)$ & 0.62 & 1.57 \\
\hline 0770 & $4.11(0.29)$ & $4.97(0.17)$ & 0.86 & $3.75(0.34)$ & $5.34(0.08)$ & 0.67 & 1.59 \\
\hline 0780 & $4.12(0.31)$ & $5.01(0.17)$ & 0.89 & $3.80(0.35)$ & $5.33(0.08)$ & 0.62 & 1.53 \\
\hline
\end{tabular}


Table 6 Single-state versus multi-state CASPT2 results (on top of the state-average CASSCF wave functions) obtained with the aug-cc-pVDZ and a series of active spaces. All the energies and coupling elements are given in $\mathrm{eV}$

\begin{tabular}{|c|c|c|c|c|c|c|c|}
\hline \multirow[t]{2}{*}{ Basis set } & \multicolumn{3}{|c|}{ Single-state CASPT2 } & \multicolumn{4}{|c|}{ Multi-state CASPT2 } \\
\hline & $1^{1} B_{u}$ & $2^{1} B_{u}$ & $\Delta \mathrm{E}$ & $1^{1} B_{u}$ & $2{ }^{1} \mathrm{~B}_{\mathrm{u}}$ & $\left|\widehat{H}_{12}^{e f f}\right|$ & $\Delta \mathrm{E}$ \\
\hline 0550 & $4.19(0.32)$ & $4.85(0.27)$ & 0.66 & $3.57(0.41)$ & $5.47(0.10)$ & 0.90 & 1.91 \\
\hline 0560 & $4.08(0.34)$ & $5.17(0.15)$ & 1.09 & $3.92(0.36)$ & $5.33(0.11)$ & 0.45 & 1.41 \\
\hline 0570 & $4.14(0.33)$ & $5.15(0.17)$ & 1.01 & $3.92(0.36)$ & $5.37(0.11)$ & 0.52 & 1.45 \\
\hline 0580 & $4.12(0.34)$ & $5.12(0.16)$ & 1.00 & $3.93(0.37)$ & $5.31(0.11)$ & 0.47 & 1.38 \\
\hline 0650 & $4.20(0.29)$ & $4.81(0.26)$ & 0.61 & $3.57(0.38)$ & $5.44(0.09)$ & 0.89 & 1.87 \\
\hline 0660 & $3.99(0.32)$ & $5.10(0.14)$ & 1.11 & $3.83(0.35)$ & $5.27(0.09)$ & 0.45 & 1.44 \\
\hline 0670 & $4.17(0.29)$ & $5.08(0.18)$ & 0.91 & $3.86(0.33)$ & $5.39(0.10)$ & 0.61 & 1.53 \\
\hline 0680 & $4.17(0.31)$ & $5.08(0.17)$ & 0.91 & $3.89(0.35)$ & $5.36(0.10)$ & 0.58 & 1.47 \\
\hline 0750 & $4.15(0.32)$ & $4.90(0.22)$ & 0.75 & $3.66(0.39)$ & $5.39(0.09)$ & 0.78 & 1.73 \\
\hline 0760 & $4.01(0.33)$ & $5.13(0.13)$ & 1.12 & $3.88(0.35)$ & $5.26(0.09)$ & 0.41 & 1.38 \\
\hline 0770 & $4.10(0.31)$ & $5.10(0.15)$ & 1.00 & $3.89(0.34)$ & $5.31(0.09)$ & 0.50 & 1.42 \\
\hline 0780 & $4.11(0.32)$ & $5.14(0.13)$ & 1.03 & $3.97(0.35)$ & $5.28(0.09)$ & 0.40 & 1.31 \\
\hline
\end{tabular}

order PT, usually underestimates it. It is entirely possible that the multi-state CASPT3 results would be more accurate that single-state ones, which would allow for explaining favorable performance of single-state CASPT2 by cancelation of errors resulting from neglecting the higher-order perturbative corrections and the off-diagonal coupling between states. Another possible explanation may come from shortcomings of our multi-state approach. Taking into account large magnitude of the off-diagonal CASPT2 coupling elements, it may be necessary to construct an effective CASPT2 Hamiltonian involving more than just two states of interest in order to describe additional static-like correlation effects in the studied wave functions. Initial results along this line show that the energy separation between the $1^{1} B_{u}$ and $2^{1} B_{u}$ states is indeed reduced upon such a modification. We are planning to present detailed account of such investigations in our next communication.

Summarizing, we have demonstrated that the multi-state CASPT2 calculations give an overall picture consistent with that obtained from single-state CASPT2 in large basis sets, unambiguously confirming existence of the substantial energy splitting between the two lowest ${ }^{1} B_{u}$ states of $2 \mathrm{~T}$. The multi-state CASPT2 results in the cc-pVDZ basis set allow us to demonstrate that the incidental quasidegeneracy of the $1^{1} B_{u}$ and $2^{1} B_{u}$ states communicated earlier by Rubio et al. originates from neglecting unusually large off-diagonal CASPT2 coupling between these two states. In general, the changes introduced by the multi-state CASPT2 procedure are too large to be regarded as small corrections to the single-state results and seem to account for new physics of the problem. The counterintuitive, better performance of the single-state CASPT2 formalism is highlighted, and possible explanations for this behavior are offered.

\subsection{Comparison with alternative methods}

The modern quantum chemistry offers a number of other approaches capable of estimating the $\mathrm{X}^{1} A_{g} \rightarrow 1^{1} B_{u}$ and $\mathrm{X}^{1} A_{g} \rightarrow 2{ }^{1} B_{u}$ excitation energies and the corresponding oscillator strengths. Table 7 gives a comparison of such data obtained using the following quantum chemical methods: time-dependent density functional theory (TDDFT [38]) with the B3LYP exchange-correlation functional, approximate single and double coupled clusters (CC2 [39]), equation-of-motion single and double coupled clusters (EOM-CCSD [40]), as implemented in the Turbomole 6.1 [41] and Molpro 2010 [42] packages. The calculations were performed using the molecular geometries optimized at the MP2 level of theory with the augcc-pVDZ and aug-cc-pVTZ basis sets. In all the wave function-based methods (MP2, CC2, EOM-CCSD), the 18 core orbitals $(1 \mathrm{~s}, 2 \mathrm{~s}$, and $2 \mathrm{p}$ orbitals for sulfur, and $1 \mathrm{~s}$ for carbons) were kept frozen after the HF stage. All the remaining orbitals were included in the correlated calculations. The results thus obtained are compared to the best single-state CASPT2 results from the current study, as well as to the experimental findings. We also attempted to account for dynamic correlation by performing the MRCISD(Q) $[43,44]$ calculations (with the Pople correction for size-extensivity [45]) on top of the 0560/aug-cc-pVDZ CASSCF wave functions. Owing to the size of the system, for each of the states in question $\left(\mathrm{X} A_{g}, 1^{1} B_{u}\right.$, and $\left.2^{1} B_{u}\right)$ the most important of the configurations constituting their CASSCF wave functions were selected for the reference 
Table 7 Comparison of the results of our alternative calculations, the best CASSCF/CASPT2 results from this study, and the experimental findings

\begin{tabular}{|c|c|c|c|c|c|c|}
\hline \multirow[t]{2}{*}{ Method } & \multirow[t]{2}{*}{ Basis set } & \multicolumn{2}{|l|}{$1{ }^{1} \mathrm{~B}_{\mathrm{u}}$} & \multicolumn{2}{|l|}{$2{ }^{1} \mathrm{~B}_{\mathrm{u}}$} & \multirow[t]{2}{*}{$\Delta \mathrm{E}[\mathrm{eV}]$} \\
\hline & & $E_{\text {exc. }}[\mathrm{eV}]$ & $f$ & $E_{\text {exc. }}[\mathrm{eV}]$ & $f$ & \\
\hline \multirow[t]{2}{*}{ TDDFT/B3LYP } & aug-cc-pVDZ & 3.80 & 0.38 & 5.09 & 0.07 & 1.29 \\
\hline & aug-cc-pVTZ & 3.79 & 0.37 & 5.06 & 0.07 & 1.27 \\
\hline \multirow[t]{2}{*}{$\mathrm{CC} 2$} & aug-cc-pVDZ & 4.16 & 0.43 & 5.30 & 0.12 & 1.14 \\
\hline & aug-cc-pVTZ & 4.13 & 0.42 & 5.24 & 0.11 & 1.11 \\
\hline \multirow[t]{2}{*}{ EOM-CCSD } & aug-cc-pVDZ & 4.32 & 0.40 & 5.40 & 0.14 & 1.08 \\
\hline & aug-cc-pVTZ & 4.36 & 0.39 & 5.48 & 0.14 & 1.12 \\
\hline \multirow[t]{2}{*}{ CASSCF/CASPT2 } & aug-cc-pVDZ & 4.12 & 0.31 & 5.01 & 0.17 & 0.89 \\
\hline & aug-cc-pVTZ & 4.11 & 0.32 & 5.14 & 0.13 & 1.03 \\
\hline MR-CISD(Q) & aug-cc-pVDZ & 4.57 & 0.27 & 5.57 & 0.06 & 1.00 \\
\hline Exp. & & 4.19 & 0.29 & 5.08 & 0.13 & 0.89 \\
\hline
\end{tabular}

space based on their CI coefficients, with the threshold value of 0.055 . We used the same set of core orbitals as in other wave function-based methods. All the MR-CI calculations were carried out with the MOLPRO 2010 package.

The common feature for all the employed techniques is a considerable energy splitting between the two ${ }^{1} B_{u}$ states in question in contrast to the previous findings of Rubio et al. The largest energy gap, exceeding $1.25 \mathrm{eV}$, is obtained in the DFT calculations. The coupled cluster methods yield a smaller value of $1.08-1.15 \mathrm{eV}$, much closer to our best single-state CASPT2 results. Moreover, the MR-CI results again demonstrated that dynamic correlation is vital in reproducing the correct energy gap between the two lowest ${ }^{1} B_{u}$ states of $2 \mathrm{~T}$, which after the MRCI treatment became considerably larger than the corresponding CASSCF value $(0.30 \mathrm{eV}$, see Table 2 for details).

Remarkably, the CASPT2/CASSCF excitation energies and intensities are in better agreement with experiment than the coupled cluster or MR-CI results. As we discussed in Sect. 4.2, the agreement may be somewhat fortuitous. However, an excellent agreement with experiment has been achieved also for the energy difference between the states, which should be less sensitive to the approximations mentioned above, owing to at least partial cancellation of errors.

\section{Discussion}

The analysis of the CASPT2 results clearly shows that the presence of a single additional orbital of the $a_{u}$ symmetry in the active space is one of the key factors responsible for the correct reproduction of the energy gap between the two ${ }^{1} B_{u}$ states and the corresponding intensity pattern. The relevant changes originate mainly from the altered description of the $2{ }^{1} B_{u}$ state, as the properties of the $1{ }^{1} B_{u}$ state are relatively unaffected by the modifications of the active space. The extension of the active space from 0550 to 0560 lowers the CASSCF excitation energy of the $2{ }^{1} B_{u}$ state by $0.17-0.18 \mathrm{eV}$. More spectacular are the corresponding changes at the CASPT2 level, where the $\mathrm{X}{ }^{1} A_{g} \rightarrow 2{ }^{1} B_{u}$ excitation energy is amplified by $0.32-0.36 \mathrm{eV}$. In consequence, the calculated energy separation between the $1{ }^{1} B_{u}$ and $2{ }^{1} B_{u}$ states is brought closer to the experimental value. Analogous effect is observed for the oscillator strength of the $\mathrm{X}^{1} A_{g} \rightarrow 2{ }^{1} B_{u}$ transition, which is reduced by over $40 \%$ becoming similar to experiment. Another important aspect of including single additional orbital of the $a_{u}$ symmetry in the conventional 0550 active space is the dramatic reduction (26-50\%) of the off-diagonal CASPT 2 coupling between the ${ }^{1} B_{u}$ states, which again brings the corresponding multi-state CASPT2 energy splitting much closer to the experimental value.

The 6th $a_{u}$ orbital is considerably more diffused than the other active orbitals, but it has no distinct Rydberg character. We verified this by adding the Rydberg basis functions generated according to the standard procedure [13, 46] to the cc-pVDZ and aug-cc-pVDZ basis sets and repeating the calculations for several of the extended active spaces studied in this work. In none of the cases, we obtained a sizable Rydberg contribution in either of the two investigated excited states, which demonstrates that the two lowest ${ }^{1} B_{u}$ states of $2 \mathrm{~T}$ have clear valence character.

Perhaps the most interesting aspect of the current study is explaining the role of the 6th orbital of the $a_{u}$ symmetry. As discussed above, its presence in the active space brings about dramatic changes to the CASPT2 energies. It is thus somewhat puzzling that this orbital remains virtually empty-its occupation number hardly exceeds 0.02 . On the other hand, the occupation numbers of other valence 
Fig. 4 Orbital occupation numbers obtained from the augcc-pVDZ CASSCF calculations for the $1{ }^{1} B_{u}$ and $2{ }^{1} B_{u}$ excited states of bithiophene using the 0550, 0560, 0570, and 0580 active spaces. The state-specific and state-averaged results for ${ }^{1}{ }^{1} B_{u}$ are denoted with multiple sign and circle, respectively, and the state-averaged results for $2{ }^{1} B_{u}$, with diamond. The occupation numbers for the additional, non-valence $a_{u}$ orbitals were negligible in all calculations and equal to $0.017-0.022$ (6th $\left.a_{u}\right)$, $0.005-0.008$ (7th $\left.a_{u}\right)$, and $0.005-0.006$ (8th $a_{u}$ )
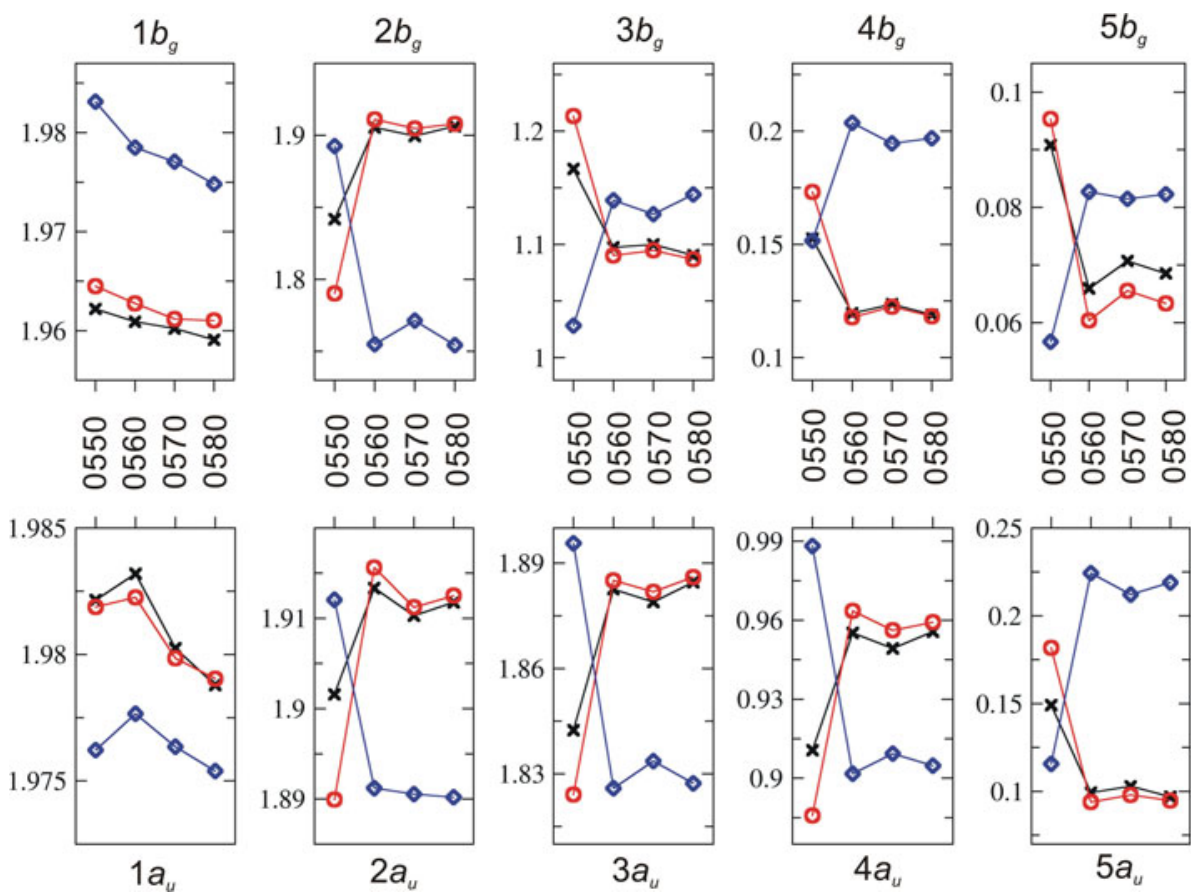
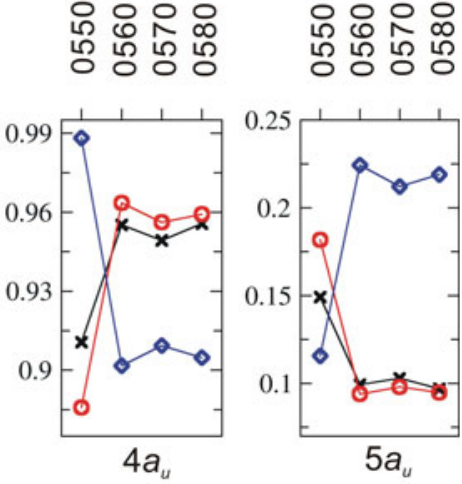

orbitals undergo considerable changes upon including the additional $a_{u}$ orbital in the active space (for details, see Fig. 4.). It is clear that extending the valence active space results in significant rearrangement of the active orbitals occupation pattern; the effect being particularly strong when the first orbital of the $a_{u}$ symmetry is added $(0550 \rightarrow 0560)$. Apparently, the function of the 6th $a_{u}$ orbital is to provide additional variational flexibility important for improving the description of the CASSCF orbitals and wave functions. We have verified this conjecture by taking the CASSCF orbitals from the stateaverage 0560/aug-cc-pVDZ calculations and using them in the CASPT2 calculations employing the following reference wave functions:

a. the 0550 CAS-CI reference wave function with the 6 th $a_{u}$ orbital entirely deleted from the orbital space;

b. the 0550 CAS-SCF reference wave function with the 6th $a_{u}$ orbital entirely deleted from the orbital space; c. the $0550 \mathrm{CAS}-\mathrm{CI}$ reference wave function with the 6th $a_{u}$ orbital shifted to the virtual orbital subspace;

d. the 0550 CAS-SCF reference wave function with the 6th $a_{u}$ orbital shifted to the virtual orbital subspace and kept frozen.

The results are given in Table 8 . One can easily see that the effects of orbital optimization are marginal. Moreover, the CASPT2 energies and transition intensities are very similar to those obtained with the 0560 active space. The CASSCF energies obtained with the 0550 active space are some $0.2 \mathrm{eV}$ higher for both states, but this shift is compensated in the subsequent CASPT2 treatment. Interestingly, including the single 6th $a_{u}$ orbital into the virtual subspace (cases $\mathrm{c}$ and $\mathrm{d}$ ) resulted in a sizable increase $(0.15-0.18 \mathrm{eV})$ of the CASPT2 corrections, which again indicates the importance of this particular orbital. The energy gap, however, is only marginally reduced with respect to the 0560 results. It should be stressed here that if

Table 8 CASSCF/CASPT2 energies [eV] and oscillator strengths from the test calculations (for details see text), using the orbitals obtained in the full 0560/aug-cc-pVDZ state-average CASSCF calculations

\begin{tabular}{|c|c|c|c|c|c|c|c|c|}
\hline \multirow[t]{2}{*}{ Case } & \multicolumn{3}{|l|}{$1^{1} \mathrm{~B}_{\mathrm{u}}$} & \multicolumn{3}{|l|}{$2^{1} \mathrm{~B}_{\mathrm{u}}$} & \multirow{2}{*}{$\begin{array}{l}\Delta \mathrm{E} \\
\mathrm{CASSCF}\end{array}$} & \multirow{2}{*}{$\begin{array}{l}\Delta \mathrm{E} \\
\mathrm{CASPT} 2\end{array}$} \\
\hline & $\mathrm{E}_{\mathrm{CASSCF}}$ & $\begin{array}{l}\mathrm{E}_{\mathrm{CASPT} 2} / \\
\text { (osc. strength) }\end{array}$ & $\begin{array}{l}\text { CASPT2 } \\
\text { correction }\end{array}$ & $\mathrm{E}_{\mathrm{CAS}}$ & $\begin{array}{l}\mathrm{E}_{\mathrm{CASPT} 2} / \\
\text { (osc. strength) }\end{array}$ & $\begin{array}{l}\text { CASPT2 } \\
\text { correction }\end{array}$ & & \\
\hline $\mathrm{a}$ & 5.72 & $4.13(0.33)$ & 1.59 & 6.07 & $5.03(0.22)$ & 1.04 & 0.35 & 0.90 \\
\hline $\mathrm{b}$ & 5.74 & $4.13(0.34)$ & 1.61 & 6.07 & $5.04(0.22)$ & 1.03 & 0.33 & 0.91 \\
\hline $\mathrm{c}$ & 5.74 & $4.00(0.33)$ & 1.74 & 6.07 & $4.85(0.22)$ & 1.22 & 0.33 & 0.85 \\
\hline $\mathrm{d}$ & 5.72 & $3.94(0.32)$ & 1.78 & 6.07 & $4.79(0.22)$ & 1.28 & 0.35 & 0.85 \\
\hline Active space: 0560 & 5.54 & $4.10(0.31)$ & 1.44 & 5.84 & $5.00(0.19)$ & 0.83 & 0.30 & 0.90 \\
\hline
\end{tabular}


the 6th $a_{u}$ orbital was allowed to mix with the other orbitals in the CASSCF calculations, the results would become identical to those obtained with the original 0550 active space. The presented analysis demonstrates that the major role of the 6 th $a_{u}$ orbital in the active space is to assist in shaping up the valence active orbitals into the correct form.

Examples of such a situation, in which adding additional orbitals to the valence active space is required for obtaining, correct results have been previously reported for oligoenes [47], as well as for 5-membered heterocyclic rings $[6,11]$. Our analysis suggests that it may be sufficient to extend the original active space using just a single additional (non-valence) orbital, which can thus be regarded as a key ingredient for constructing an appropriate active space. This observation may be especially important in calculations for larger systems, for which the size of the active space is often a critical issue.

\section{Conclusions}

In the present study, we systematically investigated the excitation energies and the corresponding oscillator strengths for the two lowest excited states of bithiophene, ${ }^{1} B_{u}$ and $2{ }^{1} B_{u}$. It is found that accurate description of the energetic separation between the two ${ }^{1} B_{u}$ states and of the corresponding intensity patterns requires using diffused basis sets of preferable triple- $\zeta$ quality and augmenting the traditionally used 0550 active space with additional, nonvalence $a_{u}$ orbital possessing significant diffuse character. The $1{ }^{1} B_{u}$ state is reasonably described in all but the smallest basis sets, and fairly insensitive to the composition of the active space. The description of the $2{ }^{1} B_{u}$ state is, however, very sensitive to both factors. We explicitly demonstrate that single-state CASPT2 calculations performed with a too small basis set (cc-pVDZ) yield distinctly different results than other single-state and multistate CASPT2 calculations. The single-state CASPT2/ccpVDZ results cause severe interpretational difficulties, which, most likely, can be regarded as computational artifacts. The reasons of pathological behavior of CASPT2 in small basis sets are analyzed, and an apt explanation in terms of multi-state CASPT2 formalism is given (unusually large couplings between the perturbed wave functions). In light of the current results, the previous findings of Rubio et al. concerning two almost quasi-degenerate ${ }^{1} B_{u}$ states computed with a double- $\zeta$ quality basis set seem to be erroneous. The actual splitting between the two ${ }^{1} B_{u}$ states is relatively large. Our CASPT2 results obtained with large basis sets and a variety of active spaces estimate it to be approximately $1 \mathrm{eV}$; these results are further confirmed in independent excited-state density functional and coupled cluster calculations. The best agreement with experiment was obtained in the single-state CASPT2/aug-cc-pVTZ/ 0780 calculations giving energy gap of $1.03 \mathrm{eV}$ and oscillator strengths of 0.32 , and 0.13 . The corresponding experimental values are $0.86 \mathrm{eV}, 0.29$, and 0.13 .

Acknowledgments National Science Council of Taiwan (grants NSC96-2113-M009-022 and NSC99-2113-M009-011) and Ministry of Education (MOE-ATU project) are acknowledged for financial support. We thank the National Center for High-Performance Computing for computer time.

Open Access This article is distributed under the terms of the Creative Commons Attribution Noncommercial License which permits any noncommercial use, distribution, and reproduction in any medium, provided the original author(s) and source are credited.

\section{References}

1. Bak B, Christensen D, Dixon WB, Hansen-Nygaard L, Andersen JR, Schottländer M (1962) J Mol Spectr 9:124

2. Nakatsuji H, Kitao O, Yonezawa T (1985) J Chem Phys 83:723

3. Serrano-Andrés L, Merchfi M, Nebot-Gil I, Roos BO, Fülscher M (1993) J Am Chem Soc 115:6184

4. Palmer MH, Walker IC, Ballard CC, Guest MF (1995) Chem Phys 192:111

5. Burcl R, Amos RD, Handy NC (2002) Chem Phys Lett $355: 8$

6. Pastore M, Angeli C, Cimiraglia R (2006) Chem Phys Lett 426:445

7. Cooper CD, Williamson AD, Miller JC, Compton RN (1980) J Chem Phys 73:1527

8. Rawlings DC, Davidson ER (1983) Chem Phys Lett 98:424

9. Nakano H, Tsuneda T, Hashimoto T, Hirao K (1996) J Chem Phys 104:2313

10. Palmer MH, Walker IC, Guest MF (1990) Chem Phys 238:179

11. Pastore M, Angeli C, Cimiraglia R (2006) Chem Phys Lett 422:522

12. Bendazolli GL, Bertinelli F, Palmerieri P, Brillante C, Taliani C (1978) J Chem Phys 69:5077

13. Serrano-Andres L, Merchan M, Fulscher M, Roos BO (1993) Chem Phys Lett 211:125

14. Palmer MH, Walker IC, Guest MF (1999) Chem Phys 241:275

15. Pastore M, Angeli C, Cimiraglia R (2007) Theor Chem Acc 118:35

16. Garnier F, Hajlaoui R, Yassar A, Srivastava P (1994) Science 265:1684

17. Dobalapur A, Torsi L, Katz HE (1995) Science 268:280

18. Garnier F, Hajlaoui R, El Kassmi A, Horowitz G, Laigre L, Porzio W, Armanini M, Provasoli F (1998) Chem Mater 10:3334

19. Friend RH et al (1999) Nature (London) 397:121

20. Meng H, Bao Z, Lovinger AJ, Wang B, Mujsce AM (2001) J Am Chem Soc 123:9214

21. Chadwick J, Kohler B (1994) J Phys Chem 98:3631

22. Rentsch S, Yang JP, Paa W, Birckner E, Schiedt J, Weinkauf R (1999) Phys Chem Chem Phys 1:1707

23. Bellêtete M, Leclerc M, Durocher G (1994) J Phys Chem 98:9450

24. Becker RS, de Melo JS, Maçanita AL, Elisei F (1996) J Phys Chem 100:18683

25. Rubio M, Merchán M, Orti E, Roos B (1995) J Chem Phys 102:3580

26. Rubio M, Merchán M, Pou-Amerigo R, Orti E (2003) Chem Phys Chem 4:1308

27. Camacho C, Cimiraglia R, Witek HA (2010) Phys Chem Chem Phys 12:5058 
28. Camacho C, Yamamoto S, Witek HA (2009) J Comput Chem 30:468

29. Choe Y-K, Witek HA, Finley JP, Hirao K (2001) J Chem Phys $114: 3913$

30. Takayanagi M, Gejo T, Hanazaki I (1994) J Phys Chem 98:12893

31. Duarte H, Dos Santos H, Rocha W, De Almeida W (2000) J Chem Phys 113:4206

32. Raos G, Famulari A, Marcon V (2003) Chem Phys Lett 379:364

33. Fedor A, Allis D, Korter T (2009) Vib Spectrosc 49:124

34. Karlström G, Lindh R, Malmqvist P-A, Roos BO, Ryde U, Veryazov V, Widmark P-O, Cossi M, Schimmelpfennig B, Neogrady P, Seijo L (2003) Computational Material Science 28:222

35. Dunning TH Jr (1989) J Chem Phys 90:1007

36. Kendall RA, Dunning TH Jr, Harrison RJ (1992) J Chem Phys 96:6769

37. Peterson KA, Dunning TH Jr (2002) J Chem Phys 117:10548

38. Casida ME, Jamorski C, Casida KC, Salahub DR (1998) J Chem Phys 108:4439
39. Hättig C, Weigend F (2000) J Chem Phys 113:5154

40. Korona T, Werner H-J (2003) J Chem Phys 118:3006

41. TURBOMOLE V6.1 2009, A development of University of Karlsruhe and Forschungszentrum Karlsruhe GmbH, 1989-2007, TURBOMOLE GmbH, since 2007

42. Werner H-J, Knowles PJ, Lindh R, Manby FR, Schütz M et al MOLPRO, version 2010. 1, a package of ab initio programs

43. Werner H-J, Knowles PJ (1988) J Chem Phys 89:5803

44. Knowles PJ, Werner H-J (1988) Chem Phys Lett 145:514

45. Pople JA, Binkley JS, Seeger R (1976) Int J Quantum Chem 10:1

46. Roos BO, Andersson K, Fülscher MP, Malmqvist P- $\AA$, SerranoAndrés L, Pierloot K, Merchán M (1996) Multiconfigurational perturbation theory: applications in electronic spectroscopy. In: Prigogine I, Rice SA (eds) Advances in chemical physics: new methods in computational quantum mechanics, vol XCIII:219. Wiley, New York

47. Nakayama K, Nakano H, Hirao K (1998) Int J Quant Chem 66:157 